\title{
Stye Can Lie! - A Rare Presentation of Scrub Typhus Eschar
}

\section{Dinesh Kumar Narayanasamy ${ }^{1}$ - Thirunavukkarasu Arun Babu ${ }^{1}$}

Received: 1 February 2020 / Accepted: 9 April 2020 / Published online: 3 June 2020

(C) Dr. K C Chaudhuri Foundation 2020

A five years old boy hailing from a rural area presented with high grade continuous fever and right eyelid swelling for $7 \mathrm{~d}$. He was treated elsewhere with antibiotics for suspected stye and orbital cellulitis. There were no similar complaints in family members. On examination, the right upper eyelid was swollen but not tender with no evidence of congestion or discharge. A blackish scab was noted along the edge of the right upper eyelid (Fig. 1a) with ipsilateral, tender preauricular lymph node. Vision was intact. He had no rashes or any other abnormalities in general and systemic examination and vitals were stable. In view of fever for $7 \mathrm{~d}$, hemogram, blood culture and serological testing for typhoid and scrub typhus was performed. IgM ELISA for Scrub typhus was positive (OD: 2.16 ) and he was promptly treated with oral Doxycycline $(2.2 \mathrm{mg} / \mathrm{kg} /$ dose, twice a day for $7 \mathrm{~d})$. Within
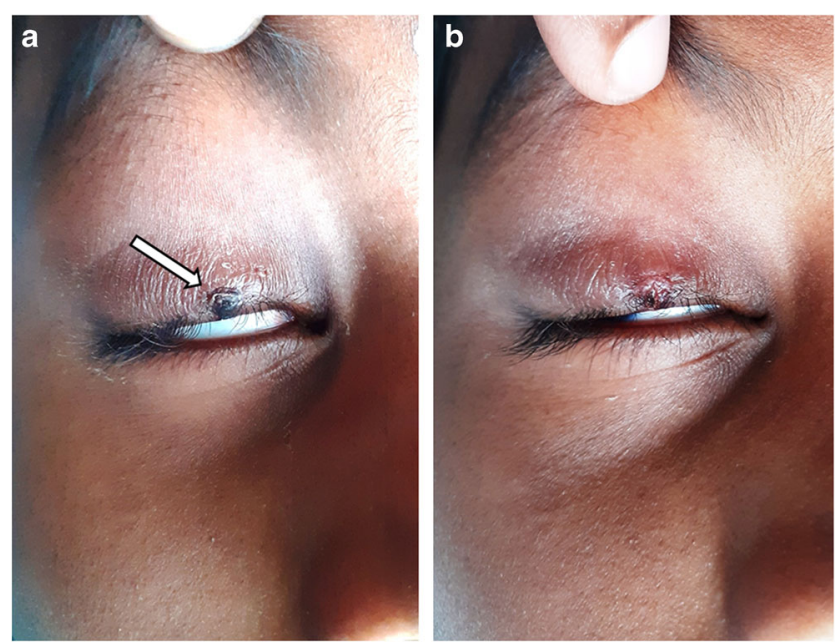

Fig. 1 a Eschar with black scab in the upper eyelid margin with lid oedema. b Eschar after falling of black scab

Thirunavukkarasu Arun Babu babuarun@yahoo.com

1 Department of Pediatrics, All India Institute of Medical Sciences (AIIMS), Mangalagiri, Andhra Pradesh 522 503, India
$48 \mathrm{~h}$ of starting the therapy, fever touched baseline and the black eyelid scab fell off (Fig. 1b). Based on the clinical presentation and positive serology, the black scab was confirmed as a typical 'Eschar' seen in scrub typhus.

Though occurrence of facial eschar in pediatric scrub typhus is rare, it is often associated with regional lymphadenopathy $[1,2]$. Wearing full clothing while playing in bushy areas, using ground mat while sitting or lying on grass and avoiding barefoot walking are simple personal protective measures to prevent scrub typhus [3]. Eschar should be considered as a differential for unilateral lid lesions, apart from the common causes like chalazion and stye (hordeolum), particularly in children presenting with fever and regional lymphadenopathy from endemic areas.

Contributions DKN and TA conceived the concept, managed the case, reviewed the literature and drafted the manuscript. Both authors have approved the final version of the manuscript. TA shall act as guarantor of the paper.

\section{Compliance with Ethical Standards}

Conflict of Interest None.

\section{References}

1. Arun Babu T, Vijayadevagaran V, Ananthakrishnan S. Characteristics of pediatric scrub typhus eschar in south Indian children. Pediatr Dermatol. 2016;34:124-7.

2. Narayanasamy D, Arunagirinathan A, Kumar R, Raghavendran V. Clinico-laboratory profile of scrub typhus - an emerging rickettsiosis in India. Indian J Pediatr. 2016;83:1392-7.

3. Sharma PK, Ramakrishnan R, Hutin YJ, et al. Scrub typhus in Darjeeling, India: opportunities for simple, practical prevention measures. Trans R Soc Trop Med Hyg. 2009;103:1153-8.

Publisher's Note Springer Nature remains neutral with regard to jurisdictional claims in published maps and institutional affiliations. 